\title{
A four-hours long burst from Serpens $X-1$
}

\author{
R. Cornelisse ${ }^{1,2}$, E. Kuulkers ${ }^{1,2}$, J. J. M. in 't Zand ${ }^{2,1}$, F. Verbunt ${ }^{2}$, and J. Heise ${ }^{1}$ \\ 1 SRON National Institute for Space Research, Sorbonnelaan 2, 3584 CA Utrecht, The Netherlands \\ 2 Astronomical Institute, Utrecht University, PO Box 80000, 3508 TA Utrecht, The Netherlands \\ Received 4 September 2001 / Accepted 8 November 2001
}

\begin{abstract}
During a serendipitous observation of the BeppoSAX Wide Field Cameras, a very long type I X-ray burst was observed from the low mass X-ray binary Serpens X-1. The burst lasted for approximately 4 hours and had an exponential decay time of $69 \pm 2 \mathrm{~min}(2-28 \mathrm{keV})$. The bolometric peak-luminosity is $(1.6 \pm 0.2) \times 10^{38} \mathrm{erg} \mathrm{s}^{-1}$ and the fluence $(7.3 \pm 1.4) \times 10^{41} \mathrm{erg}$. The first "normal" type I burst was observed 34 days after the superburst. This is in rough agreement with recent predictions for unstable carbon burning in a heavy element ocean.
\end{abstract}

Key words. accretion, accretion disks - binaries: close - stars: individual: Serpens $\mathrm{X}-1$ - stars: neutron X-rays: bursts

\section{Introduction}

Since the first report of a very long thermo-nuclear X-ray burst in 4U 1735-44 (Cornelisse et al. 2000), six more of these so-called 'superbursts' have been noted (Strohmayer 2000; Heise et al. 2000; Wijnands 2001; Kuulkers 2001). The superbursts have the following common properties: a long duration of a few hours, a large burst energy ( $\sim 10^{42} \mathrm{erg}$ ) and a persistent pre-burst luminosity between 0.1 an 0.3 times the Eddington limit $L_{\text {Edd }}$ (Wijnands 2001). In addition, all superbursts are known type I X-ray bursters.

Apart from its duration, a superburst shows all the characteristics of a type I X-ray burst, namely: the lightcurve has a fast rise and exponential decay; spectral softening occurs during the decay; black-body radiation describes the burst X-ray spectrum best. Normal type I bursts can be explained very well by unstable He and/or $\mathrm{H}$ fusion on a neutron star surface (for reviews see e.g. Lewin et al. 1993, 1995; Bildsten 1998). In contrast, the superbursts are possibly due to unstable carbon fusion in layers at larger depths than where a typical type I burst occurs (Cumming \& Bildsten 2001; Strohmayer \& Brown 2001).

In this paper we report the detection of one of the seven superbursts, namely from the X-ray source Serpens X-1 (Ser X-1), as observed with one of the Wide Field Cameras (WFC) onboard BeppoSAX. Ser X-1 is a relatively bright persistent X-ray source discovered in 1965 (Friedmann et al. 1967). Over 100 "normal" type I bursts have been reported from Ser X-1 (e.g. Swank et al. 1975; Sztajno et al. 1983; Balucińska 1985). The proposed optical counterpart

Send offprint requests to: R. Cornelisse,

e-mail: R.Cornelisse@sron.nl is MM Ser (Thorstensen et al. 1980). Wachter (1997) showed that the object is a superposition of two stars, and that no clear period could be derived from a photometric study. A distance of $8.4 \mathrm{kpc}$ derived from type I bursts is given by Christian \& Swank (1997).

In this paper we describe the observation and properties of the Ser X-1 superburst, and discuss this in context to the other superbursts reported so far. (The occurrence of this burst was first mentioned in Heise et al. 2000.)

\section{Observations}

The Wide Field Cameras are two identical coded mask cameras onboard the Italian-Dutch satellite BeppoSAX (Jager et al. 1997; Boella et al. 1997). An overview of the characteristics of the WFC is given in Jager et al. (1997).

Most WFC observations are done in secondary mode. These are arbitrary sky-pointings except that they are perpendicular to the direction of the target to which the Narrow Field Instruments onboard BeppoSAX are pointed, and dictated by solar constraints. During the first half of 1997, the WFC observed Ser X-1 for a total of $411 \mathrm{ks}$ (corrected for earth occultation and south Atlantic anomaly passages), distributed over 12 observations. In Table 1 an overview of all these observations is given.

During this period, there were also two RXTE Proportional Counter Array (RXTE/PCA) observations. The RXTE/PCA is an array of 5 co-aligned Proportional Counter Units (PCU). In Jahoda et al. (1996) a detailed description is given of the instrument. All PCU's were on during the observations. We use standard 1 data for our analysis. Also on-board RXTE are three Scanning Shadow Cameras with a $6^{\circ} \times 90^{\circ}$ field of view forming the All-Sky 
Table 1. Overview of the WFC and PCA observations of Ser X-1 between February 23 and May 13 1997. The exposure time is corrected for earth occultation, South Atlantic Anomaly passages and other data gaps. The WFC observation of Ser X1 prior to this period was on November 61996 (MJD 50393) and the one following on August 221997 (MJD 50682).

\begin{tabular}{cccc}
\hline & start (MJD) & end (MJD) & net exposure (s) \\
\hline WFC & 50502.29 & 50503.37 & 43437 \\
& 50506.82 & 50508.02 & 32237 \\
& 50508.61 & 50509.29 & 31180 \\
& 50513.16 & 50513.86 & 29020 \\
& 50517.70 & 50518.00 & 15306 \\
& 50518.26 & 50519.36 & 33699 \\
& 50529.63 & 50530.20 & 15496 \\
& 50541.26 & 50542.08 & 36302 \\
& 50542.63 & 50543.37 & 33310 \\
& 50554.62 & 50555.77 & 60707 \\
& 50563.69 & 50565.86 & 75693 \\
& 50581.47 & 50581.63 & 4652 \\
& 50535.5 & 50539.9 & 34560 \\
& 50554.4 & 50554.7 & 25920 \\
\hline
\end{tabular}

Monitor (ASM; Levine 1996). We use the data products provided by the RXTE/ASM team at the MIT web-pages.

\section{Data analysis and results}

On February 28, 1997 a flare-like event was observed which lasted for almost 4 hours. In Fig. 1 we show the RXTE/ASM lightcurve of Ser X-1 over a period of 5 years (a), and an expanded lightcurve during spring 1997 (b). The flare was observed after BeppoSAX came out of earth occultation on MJD 50507.075. In Figs. 1c and d a detailed view of the flare is shown. The rise to maximum was missed. After the satellite came out of earth occultation a flat top is observed before the decay starts, so it appears that the peak is covered. The flare shows an exponential decay. In Fig. 1e we see that spectral softening occurs during the decay. This is indicative for type I bursts. In Table 2 we summarize the exponential decay times from fits in different energy bands.

We divided the observation in which we discovered the superburst (see Figs. 1c and d) in three different intervals before, during and after the superburst, as indicated by dashed lines in Fig. 1c. In the first and last interval we fit the persistent flux with solely an absorbed bremsstrahlung spectrum. During the superburst we used a sum of the (persistent) bremsstrahlung and black-body radiation to describe the flux, taking the persistent bremsstrahlung emission as fixed at the average level of the spectral fits before and after the burst. We tried several other spectral models for the persistent emission, like a cut-off powerlaw and a disk black-body. The derived fluxes did not change significantly for the different models, and the best fit during the superburst is given by the bremsstrahlung model. In all our fits we fixed the absorption column at $N_{\mathrm{H}}=0.5 \times 10^{22}$ atoms cm ${ }^{-2}$ (Christian \& Swank 1997).
Table 2. Fit results of the superburst. The top panel shows the decay times for exponential decay fits in different passbands. The next panels shows the spectral fit results for the persistent emission before, after and during the burst, respectively. A bremsstrahlung model is employed to describe the persistent emission. The burst-emission is described by a sum of the bremsstrahlung and black-body spectrum, taking the bremsstrahlung emission fixed at the average of the spectral fits before and after the burst. For all spectral fits we fixed the absorption column at the value of $N_{\mathrm{H}}=0.5 \times 10^{22}$ atoms $\mathrm{cm}^{-2}$ (from Christian \& Swank 1997).

\begin{tabular}{|c|c|}
\hline \multicolumn{2}{|l|}{ exponential decay } \\
\hline$\tau_{2-28 \mathrm{keV}}(\min )\left(\chi_{\nu}^{2}\right.$, d.o.f. $)$ & $69 \pm 3(1.2,53)$ \\
\hline$\tau_{2-5 \mathrm{keV}}(\min )\left(\chi_{\nu}^{2}\right.$, d.o.f. $)$ & $108 \pm 12(1.2,51)$ \\
\hline$\tau_{5-28 \mathrm{keV}}(\min )\left(\chi_{\nu}^{2}\right.$, d.o.f. $)$ & $52 \pm 2(1.4,56)$ \\
\hline \multicolumn{2}{|l|}{ brems before } \\
\hline$k T_{\mathrm{br}}(\mathrm{keV})$ & $7.3 \pm 0.4$ \\
\hline$F_{2-28 \mathrm{keV}}\left(10^{-9} \mathrm{erg} \mathrm{cm}^{-2} \mathrm{~s}^{-1}\right)$ & $5.0 \pm 0.2$ \\
\hline$F_{\mathrm{bol}}\left(10^{-9} \mathrm{erg} \mathrm{cm}^{-2} \mathrm{~s}^{-1}\right)$ & $8.3 \pm 0.2$ \\
\hline$\chi_{\nu}^{2}$ (d.o.f.) & $0.8(27)$ \\
\hline \multicolumn{2}{|l|}{ brems after } \\
\hline$k T_{\mathrm{br}}(\mathrm{keV})$ & $6.2 \pm 0.4$ \\
\hline$F_{2-28 \mathrm{keV}}\left(10^{-9} \mathrm{erg} \mathrm{cm}^{-2} \mathrm{~s}^{-1}\right)$ & $5.7 \pm 0.3$ \\
\hline$F_{\mathrm{bol}}\left(10^{-9} \mathrm{erg} \mathrm{cm}^{-2} \mathrm{~s}^{-1}\right)$ & $10.1 \pm 0.4$ \\
\hline$\chi_{\nu}^{2}$ (d.o.f.) & $1.0(27)$ \\
\hline \multicolumn{2}{|l|}{ brems \& black-body during } \\
\hline$k T_{\mathrm{bb}}(\mathrm{keV})$ & $2.4 \pm 0.1$ \\
\hline$R(\mathrm{~km}$ at $8.4 \mathrm{kpc}$ distance $)$ & $3.6 \pm 0.3$ \\
\hline$F_{2-28 \mathrm{keV}}\left(10^{-9} \mathrm{erg} \mathrm{cm}^{-2} \mathrm{~s}^{-1}\right)$ & $6.1 \pm 1.1$ \\
\hline$F_{\text {bol }}\left(10^{-9} \mathrm{erg} \mathrm{cm}^{-2} \mathrm{~s}^{-1}\right)$ & $6.3 \pm 1.1$ \\
\hline$L_{\text {peak }}\left(10^{38} \operatorname{erg~s}^{-1}\right)$ & $1.6 \pm 0.2$ \\
\hline$E_{\mathrm{b}}\left(10^{41} \mathrm{erg}\right)$ & $7.8 \pm 1.4$ \\
\hline$\chi_{\nu}^{2}$ (d.o.f.) & $1.7(26)$ \\
\hline
\end{tabular}

We also added a black-body component to model the emission after the burst. However, an F-test showed that this extra component did not improve the fit significantly $(\simeq 2.5 \sigma$ probability)

In Fig. 2 the results of the time-resolved spectral fits are shown. It is seen that both the black-body flux and the temperature drop exponentially, while the radius stays nearly constant. This is typical for normal type I bursts.

An increase in the RXTE/ASM count-rate is observed on MJD 52120.15. This increase lasted for several dwells, having a total duration of at least $8.6 \mathrm{~min}$ and less then 2.3 hrs. Spectral softening can not be proven. This flarelike event may be another superburst.

\section{Discussion}

The flare-like event from Ser X-1 shows, except for its duration, all characteristics of a type I burst. There are now six sources showing superbursts lasting for hours up to half a day. This superburst occurred at a persistent flux level of $0.21 L_{\mathrm{Edd}}$, for $L_{\mathrm{Edd}}=2 \times 10^{38} \mathrm{erg} \mathrm{s}^{-1}$. This is comparable to that for the other superbursts. The fact that the persistent luminosity before the superburst is similar in the 6 sources may be an observational selection effect. 

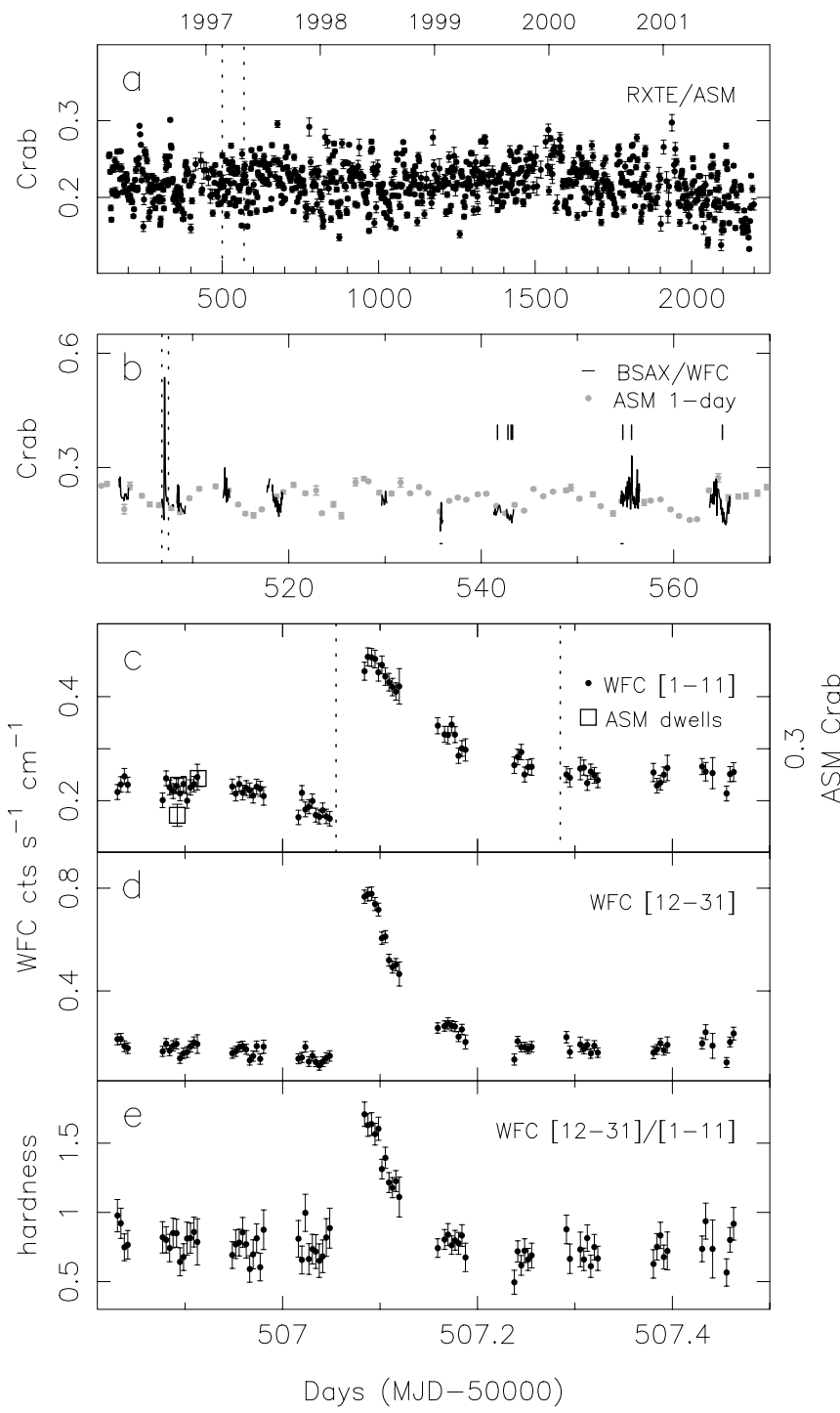

Fig. 1. a) RXTE/ASM lightcurve of Ser X-1. Each bin is a 2 day average, and normalized to that of the Crab; only data with more than one dwell per day were included. The time span between the dashed lines has been blown up in $\mathbf{b}$ ), where we also show the data of the WFC (2-28 keV), and RXTE/PCA (indicated by small horizontal bars underneath). The WFC bins are one orbit averages and the PCA bins are 5 min averages; data points are connected to guide the eye when less than 0.2 days apart. The ASM points are one day averages (with more than one dwell per day). All observations are normalized to the Crab. A sharp increase can be seen at MJD 50507. The vertical tick-marks at the middle-right indicate the occurrence of "normal" type I bursts. Panels c)-e) show again a blow-up, indicated by the dashed line in $\mathbf{b}$ ), of the superburst in two different energy-bands, i.e. $2-5 \mathrm{keV} \mathrm{c}$ ) and $5-28 \mathrm{keV} \mathrm{d}$ ), and their ratio in e). Each bin is 5 min. In panel c) individual ASM dwells are also over-plotted.

As suggested by Cumming \& Bildsten (2001), superbursts can occur at higher luminosities, where they are more difficult to detect due to the smaller contrast with the persistent flux. The total energy emitted during the burst,

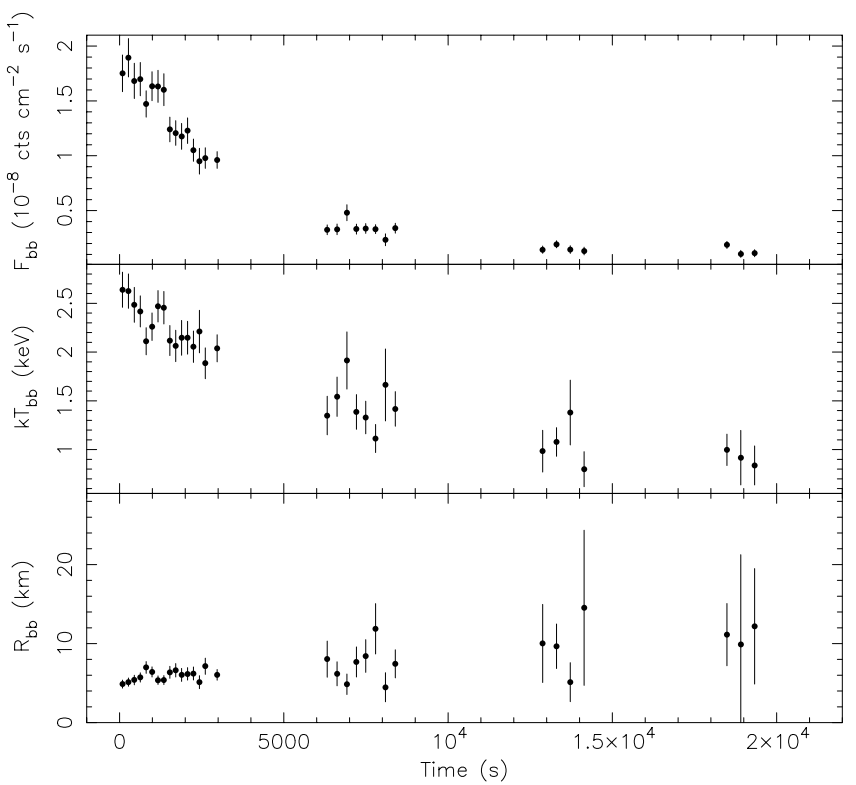

Fig. 2. Results from time resolved spectroscopy from the beginning of the burst back to the persistent emission level. We show the black-body flux $\left(F_{\mathrm{bb}}\right)$, black-body temperature $\left(k T_{\mathrm{bb}}\right)$ and black-body radius $\left(R_{\mathrm{bb}}\right)$ of the burst, respectively. We have taken the persistent emission level, modeled by a bremsstrahlung spectrum, as fixed at the same values as used with the spectral fit of the total burst (see Table 2). Also the absorption column is fixed at $N_{\mathrm{H}}=0.5 \times 10^{22}$ atoms cm $^{-2}$ (Christian \& Swank 1997). During the first part of the burst each bin is $180 \mathrm{~s}$. After the first gap due to earth occultation the bin size becomes $300 \mathrm{~s}$ and the last two intervals have bin sizes of $420 \mathrm{~s}$. The black body radius is for a source at $8.4 \mathrm{kpc}$ distance.

$E_{\mathrm{b}}=(7.3 \pm 1.4) \times 10^{41} \mathrm{erg}$, and the exponential decay time, are also comparable to those of the other superbursts.

Cornelisse et al. (2000) noticed the absence of normal type I bursts in the regular burster $4 \mathrm{U} 1735-44$ throughout the 9-day observation. In the case of KS1731-260, no type I bursts were seen after the superburst, whereas they were present beforehand (Kuulkers et al. 2001). From Fig. 1 we see a similar effect in Ser X-1. The first normal burst is observed about 34 days after the superburst, at MJD 50541.32. After that, Ser X-1 is seen to burst rather regularly, having a total of seven bursts in $210.7 \mathrm{ks}$ (about 3 bursts per day). For this burst-rate the Poisson distribution predicts a probability of $24 \%$ that no bursts are detected in the $55 \mathrm{ks}$ WFC observations before the superburst, so it is possible that the lack of observed bursts before the superburst is due to chance. The probability that the absence of ordinary bursts in the 34 days after the superburst is due to chance is $1.2 \%$.

We also performed Monte Carlo simulations to verify in more detail the significance of finding no bursts around the superburst. We randomly varied the burst waiting time in an interval symmetric around 0.38 day (i.e. the average waiting time), and taking as the lower limit 0.04 day (the lowest waiting time observed by Sztajno et al. 1983). 
We then determined the number of bursts in: 1) the observing window before the superburst, and 2) the observing windows between the superburst and the occurrence of the first normal type I burst. By doing $10^{6}$ simulations for each window we found that the expected number of bursts for window 1) is $1.5 \pm 1.1$ and the chance of observing no bursts is $14 \%$. For window 2) the expected number of bursts is $4.9 \pm 1.9$ and the chance of observing no bursts is only $0.4 \%$. We conclude that the absence of bursts after the superburst is significant.

Figure 1 shows that after the superburst the persistent flux-level in the low energy passband is higher then before the burst, while in the high energy passband no significant increase is observed. This is also visible in the burst from $4 \mathrm{U} 1735-44$ (Cornelisse et al. 2000), but less obvious in KS 1731-260 (Kuulkers et al. 2001). This offset could be due to the heating of the neutron star atmosphere after the superburst, and could be present in the spectrum as a black-body component. However, for both Ser X-1 and KS 1731-260 no significant blackbody contribution can be proven. We re-analyzed the offset emission after the superburst of $4 \mathrm{U} 1735-44$ (interval MJD 50318.55-50319.0). Here, $\chi^{2}=38.2$ (27 d.o.f.) for the absorbed bremsstrahlung model, which improves to $\chi^{2}=17.2$ (25 d.o.f.) when a black-body component was added. Performing an F-test shows that this is a significant ( $\simeq 4 \sigma$ probability) improvement. This may indicate that, at least for $4 \mathrm{U} 1735-44$, the neutron star atmosphere is still cooling $\simeq 0.5$ day after the superburst.

Cumming \& Bildsten (2001) propose that these bursts are due to unstable carbon burning in an ocean of heavy elements, e.g. ${ }^{104} \mathrm{Ru}$. This can explain the observed durations and fluences for these superbursts. Also, the recurrence time is roughly consistent with that found for $4 \mathrm{U} 1636-53$ (Wijnands 2001). A waiting time is predicted before the normal type I bursts start again; $t_{\text {wait }} \simeq 5 t_{\text {cool }}$, where the cooling time is a function of mass accretion rate (Cumming \& Bildsten 2001). In the case of Ser X-1, for which the persistent flux is roughly $0.2 L_{\mathrm{Edd}}$, a waiting time of $\sim 15$ days is predicted. Given the rough estimates above, and the small observational coverage between 15 days and 34 days after the burst, the predicted waiting time is consistent with the observation.

If the increase in the RXTE/ASM data is due to a superburst, an upper limit of 4.4 years on the recurrence time of superbursts in Ser X-1 can be estimated.
This timescale is comparable to the 4.7 years found for 4U 1636-53 (Wijnands 2001).

Acknowledgements. We thank Lars Bildsten for critically reading the manuscript, and Rudy Wijnands for pointing out the existence of the RXTE/ASM flare. The BeppoSAX satellite is a joint Italian and Dutch program. We made use of the quicklook results provided by the RXTE/ASM team.

\section{References}

Balucińska, M., \& Czerny, M. 1985, AcA, 35, 291

Bildsten, L. 1998, in The many faces of neutron stars, ed. J. Bucheri, J. van Paradijs, \& M. A. Alpar (Kluwer, Dordrecht), 419

Boella, G., Butler, R., Perola, G., et al. 1997, A\&AS, 122, 299

Christian, D. J., \& Swank, J. H. 1997, ApJS, 109, 177

Cornelisse, R., Heise, J., Kuulkers, E., et al. 2000, A\&A, 357, L21

Cumming, A., \& Bildsten, L. 2001, ApJ, 559, L127

Friedmann, H., Byram, E., \& Chubb, T. 1967, Science, 156, 374

Heise, J., In 't Zand, J. J. M., \& Kuulkers, E. 2000, HEAD, $32,28.03$

In 't Zand, J. J. M. 2001, in Proc. 4th INTEGRAL workshop (Sep. 2000, Alicante, Spain), ed. V. Reglero, in press [astro-ph 0104299]

Jager, R., Mels, W., Brinkman, A., et al. 1997, A\&AS, 125, 557

Jahoda, K., Swank, J. H., Stark, M. J., et al. 1996, Proc. SPIE, 2808,59

Kuulkers, E. 2001, ATEL \#68

Kuulkers, E., In 't Zand, J. J. M., Kerkwijk, M. H., et al. 2001, submitted to A\&A

Levine, A. M., Bradt, H., Cui, W., et al. 1996, ApJ, 469, L33

Lewin, W., van Paradijs, J., \& Taam, R. 1993, Space Sci. Rev., 62,223

Lewin, W., van Paradijs, J., \& Taam, R. 1995, in X-ray binaries, ed. W. Lewin, J. van Paradijs, \& E. van den Heuvel (Cambridge U.P., Cambridge), 175

Strohmayer, T. E. 2000, HEAD, 32, 24.10

Strohmayer, T. E., \& Brown, E. F. 2001, ApJ, submitted [astro-ph/0108420]

Swank, J., Becker, R., Pravdo, S., et al. 1976, IAUC, 2963

Sztajno, M., Basinska, E. M., Cominsky, L. R., et al. 1983, ApJ, 267, 713

Thorstensen, J. R., Charles, P. A., \& Bowyer, S. 1980, ApJ, 238,964

Wachter, S. 1997, ApJ, 490, 401

Wijnands, R. 2001, ApJ, 554, L59 